\title{
Field Dependency and the Sense of Object-Presence in Haptic Virtual Environments
}

\author{
DAVID HECHT, M.A. ${ }^{1,2}$ and MIRIAM REINER, Ph.D. ${ }^{1,3}$
}

\begin{abstract}
Virtual environment (VE) users often report having a sense of being present in the virtual place or a sense that the virtual object is present in their environment. This sense of presence depends on both the technological fidelity (e.g., in graphics, haptics) and the users' cognitive/ personality characteristics. This study examined the correlation between user's cognitive style on the field-dependency dimension and the level of object-presence they reported in a haptic VE. Results indicated that field-independent individuals reported higher presence ratings compared to field-dependent participants. We hypothesize that field-independents advantage in reorganizing the perceptual field and constructing it according to their previously acquired internal knowledge enables them to cognitively reconstruct the VE experience more efficiently by selectively attending only to the relevant cues and by filling in the gap of missing information with their previous knowledge and creative imagination. This active and creative cognitive process may be behind the enhanced sense of presence. In addition, we raise a possible linkage between field dependency, the sense of presence, and simulator sickness phenomenon.
\end{abstract}

\section{INTRODUCTION}

V IRTUAL ENVIRONMENT (VE) users often report having a sense of being present in the virtual place or a sense that the virtual object is present in their environment. Individual differences in cognitive abilities and in personality characteristics are factors affecting this sense of presence. However, there are relatively few studies on the relations between user's characteristics and presence. Studies based on the Neuro-Linguistic Programming model, differentiating people according to their preferred sensory channel for encoding subjective experiences and memories, suggested that visually dominated people experienced the highest sense of presence, while the auditory dominated group gave the lowest presence ratings. For kinesthetic dominated participants the results were not clear, since they reported high presence in one condition but low presence in another condition. ${ }^{1-3}$

Other studies correlated presence ratings with absorption and immersion, the tendency to become fully involved and immersed in common everyday mental activities, and found that higher immersive tendencies can predict a high sense of presence. ${ }^{4,5}$ Creative imagination, empathy, willingness/openness to new experiences and motivation/interest in the topic were also found to correlate with presence, in a way that those individuals, who are highly fantasy prone, or more empathic, creative, willing to be transported to the virtual world or more interested in the topic, experienced a greater sense of

\footnotetext{
${ }^{1}$ The Touch Laboratory, Department of Behavioral Biology, Technion-Israel Institute of Technology, Haifa, Israel.

${ }^{2}$ University of Haifa, Haifa, Israel.

${ }^{3}$ Department of Education in Technology and Science, Technion-Israel Institute of Technology, Haifa, Israel.
} 
presence in the VE. ${ }^{6-9}$ Also, people who are either introverts, scoring high in spatial intelligence, or feeling highly anxious during test situations were found to rate higher their presence experience in VE. ${ }^{10}$ This study aimed to investigate a possible relationship between the cognitive style field-dependency and the sense of presence in VE.

\section{Field dependency}

Field dependency is defined as the degree to which a person's perception is affected by the context of the surrounding perceptual field. ${ }^{11,12}$ Two standard tests measure this continuum of cognitive ability. The Rod \& Frame Test (RFT) in which participants, sitting in a dark room, are asked to rotate an illuminated rod to a vertical position, in the presence of a conflicting context-an illuminated tilted frame surrounding the rod. Field-Dependent (FD) individuals perform poorly on this test, as they take the frame as a reference point for horizontal and vertical and try to rotate the rod in relation to the frame. Whereas Field-Independent (FI) participants succeed better on the tasks' cognitive demand - to ignore the tilted frame-as they use intrinsic cues such as body posture, vestibular, and gravitation force as references. Figure 1 illustrates a typical performance of FD and FI on the RFT. Scores can be calculated by using several methods, ${ }^{13}$ but the original, most conventional and widely used is the mean degrees of absolute deviations from the true vertical of participants' rod placements.

The Embedded Figures Test (EFT) is another measure, in which participants are initially presented with a simple figure and then with a more complex figure embedding the simple figure in it. Their task is to find the simple figure as fast as possible within an allocated time ( $3 \mathrm{~min}$ per figure). FD subjects find it hard to extricate the simple figures from the complex contexts, while FI are less affected by the context and able to employ an analytic approach to the problem. Scores are calculated as the sum of the time each participant needed to find all simple figures in a series of 12 complex figures.

According to the field dependence theory, although the EFT in its strictest interpretation is a perceptual test, reflecting extent of competence at perceptual disembedding, nevertheless, it appears that the ability to overcome the organization of the field, to break it up in order to locate a sought-after component, manifests itself, in congruent form, also in other areas of the person's psychological activity, for instance, in the Rorschach inkblots. Characteristically, FD tend to leave such material "as is," rather than imposing structure on it, and the result is vague and indefinite percepts. In contrast, FI are more likely to impose structure on the inkblots which lack it, and therefore their percepts are more organized and definite. Thus, the RFT and EFT demonstrate a general difference in cognitive style when presented with incomplete, confusing or conflicting information. While FD rely heavily on external sources-the surrounding environment and its contextual cues-and they are profoundly affected by them, FI are able to ignore these external cues and rely mainly on their internal sources. ${ }^{11,14}$

Many studies support the view that differences between FD and FI are expressed, not only in perception, but rather in the entire range of cognition and behavior. In a classic study, ${ }^{15}$ a group of college students wrote their views on the use of antihistamines. Sometime later, they were asked to read a made-up passage apparently from an authoritative journal, which contradicted their previously expressed views, and they were asked to write out their views once again. FD, in defining their views on the second round, proved more likely to shift from their initially stated position to the position attributed to the authority. Similarly, FD were found to be more prone to misleading by interrogators'

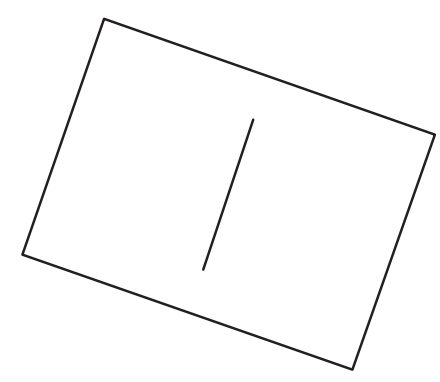

Field Dependent (FD)

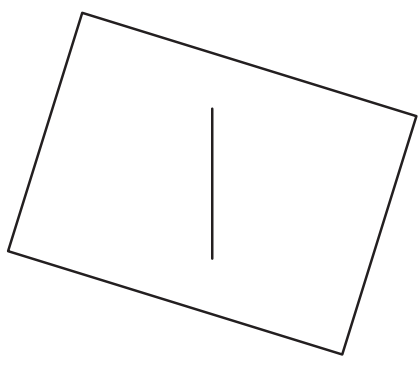

Field Independent (FI)
FIG. 1. A typical performance of FD and FI on the rod and frame test. 
suggestions attempting to reconstruct their memories of certain events. ${ }^{16,17}$ Another correlation study between field-dependency and suggestibility indicated that FD, if paired with an FI partner on the RFT, are more liable to be influenced by their partners' judgments and change their performance pattern, than FI. ${ }^{18}$ Likewise, FD are more easily hypnotized than $\mathrm{FI}_{1}{ }^{19}$ and in a study that asked participants to talk about a subject that interested them, FI produced the same amount of verbal output whether the interviewer was responsive or sat silent. However, FD showed much reduced verbal output with the silent interviewer-suggesting that, without external information, they lacked any confirmation that what they were saying was appropriate. ${ }^{20}$

FD also tend to be more sensitive to social cues and influenced by them, while FI who organize their world by themselves are more autonomous and rely mainly on internal cues. ${ }^{12,21}$ Thus, FD are more liable to answer according to social expectations. ${ }^{22}$ Similarly, the belief in paranormal phenomena is more common amongst FD. ${ }^{23}$ Field-dependency had been linked also to cultural differences as cross-cultural studies found that Westerners are relatively more FI than East Asians, ${ }^{24-27}$ a finding that correlates well with the strong emphasis in Western culture on individualism, where the self is experienced as an autonomous, independent entity, as opposed to East Asian cultures' emphasis on collectivism, where the self is perceived as fundamentally interconnected and in relationships with important others. ${ }^{28,29}$

It is important to note here, that field dependency is not a dichotomist category; it is rather a continuum (or dimension) on which all people lie. The ends of the continuum, FD and FI are relative to the sample being tested, and any sample will tend to distribute itself along a normal curve. Furthermore, the adaptive value of 'independence' depends on the setting in which an individual is required to function, whereas in some situations/ societies, the requirements of living are best met by characteristics such as independence, in other situations and cultural settings, the characteristics of relatively limited independence and differentiation may provide a better match. Therefore, in the context of field dependency, designators such as "independent" or "dependent" do not carry with them any (positive or negative) value judgments about the person to whom they are applied, since value judgments require a referent. Meaning, FI are not "superior" and FD are not "inferior." In turn, field dependency is functional and more suitable in certain tasks/situations and vice versa in other circumstances. As a very general statement, it can be said that FI are usually better in cognitive skills demanding analytical ability, while FD are better at interpersonal skills demanding a good evaluation of the social cues, ${ }^{30,31}$ but in many other situations, which may require a balance between cognitive and personal skills, it is hard to predict who will achieve better results. ${ }^{14}$

The rational for this study, on the relation between field dependency and the sense of presence in VE, was based on the following consideration. In order to feel and experience presence, it requires, to some degree, an active and creative effort from the user's side in taking the raw, impoverished and incomplete physical stimuli generated by technology and combining it together with his prior knowledge, expectations, creative imagination, motivation etc. in a way that reconstruct it into a coherent and meaningful experience that creates a "resonance" between the environmental sensory cues and past memories. ${ }^{32}$ Furthermore, it requires also paying attention mostly to the meaningful information while ignoring the irrelevant and "noisy" data. Thus, we hypothesized that FI advantage in this cognitive ability-to "reorganize the perceptual field and construct it according to their, previously acquired, internal knowledge, while ignoring irrelevant stimuli"14 — would enable them to cognitively reconstruct the VE experience more efficiently, by ignoring irrelevant stimuli and by using their internal knowledge and creative imagination to "fill in the gap" of missing information, sensations, perceptions etc. and this should lead to a greater sense of presence, compared to FD.

\section{Object-presence}

The sense of presence is not similar in all VE systems. In fully surrounding and immersive VE systems (such as HMD's and the CAVE) that capture the entire perceptual field, through a head mounted display or $360^{\circ}$ presentation, the user develops a feeling that he is actually inside a place which does not exist in the real world. Thus, the sense of presence refers to his sense of being present in a (virtual) place. Whereas in non-immersive VE systems (such as projection-tables, projectionaugmented models, haptic VE's [e.g., ReachIn ${ }^{\circledR}$ PHANTOM desktop $\left.{ }^{\mathrm{TM}}\right]$ ), although users usually wear stereo-goggles which, to a certain extent, narrows their vision to the virtual scene by blocking parts of their side-view, nevertheless, they do not have a feeling of being transformed to a different place. Hence, the sense of presence, in these VE systems, refers to the presence of a (virtual) object; i.e. the user feels that he can actually touch and manipulate an 


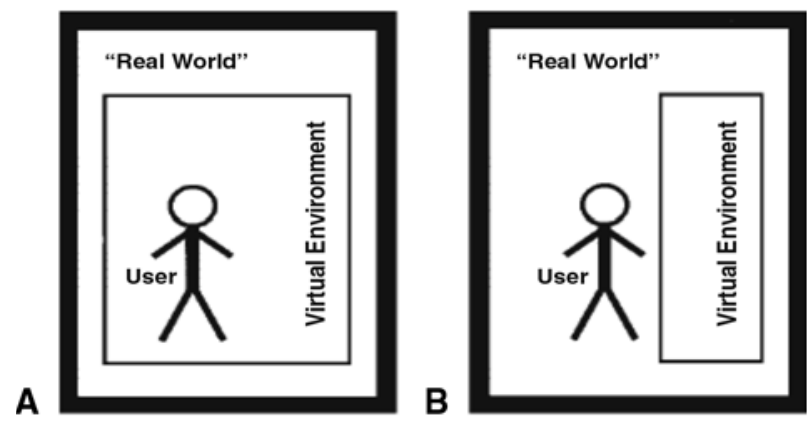

FIG. 2. (A) Fully surrounding VE systems-Presence in a place. (B) Non-surrounding systems (e.g., projection-tables, projection-augmented models, haptic VE's) - Presence of an object. (Illustration adapted from Stevens et al. ${ }^{34}$ )

object that does not exist in the real world. This distinction is elaborated and discussed more extensively elsewhere ${ }^{33,34}$ and illustrated in Figure 2.

Although a decade of research had yielded an assortment of presence measures (collected and organized at: www.presence-research.org), almost all of these tools (questionnaires, behavioral and physiological measures) were designed to measure the sense of presence in a place, except one questionnaire that had been developed to measure the Presence of an object. This ObjectPresence Questionnaire (OPQ $)^{34}$ is actually based on the general concepts of an earlier presence questionnaire $^{4}$ with some modifications to make it suitable for non-immersive haptic virtual systems, and it measures the subjective experience that a particular object exists in the user's environment, even though that object is only virtual and does not exist in the real world. Since we used in this study the non-immersive haptic VE, we measured presence with the OPQ. ${ }^{34}$

\section{METHODS}

Eighteen participants (nine males, nine females, mean age $=23.2$ years, $\mathrm{SD}=2.18$ ) were tested on the EFT. Scores reflected the time (in seconds) they needed to locate the simple figures embedded in the complex figures. Immediately afterwards, they were presented with a line of virtual objects which were developed on the ReachIn ${ }^{\circledR}$ system (PHANTOM desktop ${ }^{\mathrm{TM}}$ with haptic device by SensAble Technologies ${ }^{\odot}$ ). These virtual objects were: toothpaste, a toy-trumpet, a glue-pad, a chessboard with pieces, a globe, a telephone with press buttons, and cubes. These virtual objects were both, visible and touchable through a stylus, in a three-dimensional (3D) space.

When presented with the virtual globe, their task was to find five countries located at different places around the globe, thus in their search, participants had to use the stylus to rotate the globe (in all three axes) and feel it haptically. Touching the chessboard-pieces via the stylus, participants could see them in 3D and feel the roundness of the pawns' top, the zigzag shape of the queens' crown, and so forth, as if these virtual pieces were actually present in the real world. They were clearly told that they are not expected to win the game nor to develop any strategy but simply play a few moves freely as they desire. With the virtual telephone they were requested to dial up to a familiar telephone number, thus they used the stylus to press the dial buttons. And with the virtual cubes, they were requested to push them with the stylus towards a hit target, which required them to haptically manipulate the cubes in all three axes. The virtual toothpaste, toy-trumpet and glue-pad were haptically explored and manipulated freely with no specific instructions. Total time for this exploration, manipulation and play with all these virtual objects, was about $15 \mathrm{~min}$. Immediately afterward, participants described and reported their virtual-reality experience by answering the OPQ. ${ }^{34}$

\section{RESULTS}

Analysis of a Pearson correlation between fielddependency scores as measured on the EFT and the OPQ scores (sum of all 30 items) indicated a significant negative correlation $[r=-0.423, p=0.04]$. That is, FI participants with the lowest scores on the EFT (less time needed to find the simple figures) reported a higher sense of object-presence, and vice versa for FD. Grouping specific items from the questionnaire into four clusters: Involvement/ Control, Naturalness, Interface Quality and Haptics (clustered in accordance with the Witmer and Singer ${ }^{4} \mathrm{PQ}$ cluster analysis) revealed significant correlations between all four clusters as follows: Involvement/Control and Naturalness [ $r=0.851$, $p<0.000]$, Involvement/Control and Interface Quality [ $r=0.553, p=0.009]$, Involvement/Control and Haptics $[r=0.695, p=0.001]$, Naturalness and Interface Quality [ $r=0.449, p=0.031]$, Naturalness and Haptics [ $r=0.791, p<0.000]$, Interface Quality and Haptics [ $r=0.647, p=0.002]$. These positive correlations between the clusters indicates the strong construct validity and internal consistency 
of the OPQ that all its items test the same cognitive construct-object-presence.

We investigated further these results, correlating the EFT scores with every specific item on the OPQ using the Spearman non-parametric correlation. This analysis also revealed significant negative correlation between field-dependency and the following items of the questionnaire (numbered as in the original OPQ): (Q8) How much did your experiences with the object seem consistent with your real world experiences? [ $r=-0.442, p=0.03]$. (Q9) Were you able to anticipate what would happen next in response to the actions that you performed? $[r=-0.437, p=0.03]$. (Q10) How completely were you able to actively survey or search the object using vision? $[r=-0.468, p=0.02]$. (Q18) How involved were you with the experience of the object? $[r=-0.452, p=0.03]$. (Q30) Were there moments during the VE experience when you felt completely focused on the task or object? [ $r=-0.449, p=0.03]$.

Gender differences are consistently reported in the field dependency literature. Men tend to be, on average, more FI than women, and although the difference is usually small, it is consistent across studies. ${ }^{35}$ In this study, there was also a slight gender difference, where the average time men needed to complete the EFT was $5.05 \mathrm{~min}$, while the women needed $5.25 \mathrm{~min}$. Therefore, we further analyzed the correlations between EFT scores and every specific item on the OPQ, for each gender separately. In males $(n=9)$, there were high correlations in the following items: (Q5) How much did the haptic aspects of the object involve you? [ $r=0.676, p=0.02]$. (Q10) $[r=-0.648, p=0.03]$. (Q18) $[r=-0.822, p=0.003]$. (Q19) How much delay did you experience between your actions and expected outcomes? $[r=-0.639, p=$ 0.03]. (Q31) How easily did you adjust to the control devices used to interact with the object? $[r=-0.685$, $p=.02$ ]. In females $(n=9)$, the following items were found to correlate: (Q17) How well could you move or manipulate the object? $[r=-0.624, p=0.04]$. (Q24) How well could you concentrate on the assigned tasks or required activities rather than on the mechanisms used to perform those tasks or activities? [ $r=-0.806, p=0.004]$. As can be seen, specific items of the OPQ are highly correlated with EFT performance, differently for men and for women.

\section{DISCUSSION}

These results indicate a negative correlation between field-dependency and the sense of objectpresence. That is, FI with the lowest scores on the EFT (less time needed to find the simple figures) reported a, relatively, higher sense of objectpresence on the OPQ, and vice versa, FD with the highest scores on the EFT reported a, relatively, lower sense of object-presence.

A possible explanation for these results may be that the sense of presence, whether it is being present in a (virtual) place or the presence of a (virtual) object is a complex cognitive construct created not only by technology's advancements (e.g., in graphics, 3D presentation, haptics, synchronization), but it is the user's mind that takes these computer generated stimuli and turns it into an experience. A robot encountering a virtual precipice will not be afraid nor will it show any behavioral or physiological responses (unless programmed so) whereas a person who is exposed to the same computer generated stimuli will think, feel, behave, and respond physiologically in a certain manner. ${ }^{36}$ That is to say, sensorial realism certainly has an important influence on presence, but there is much more to the story, since even perfectly mediated sensory perception would not by itself automatically induce a strong sense of presence. Presence as a subjective experience is rather an active and creative process of the user's mind that perceives the computergenerated raw stimuli, and turns it, with all his mental (cognitive and emotional) capacities, into an experience of presence. Thus, just as there is no perception without a perceiver, in VE, there is no sense of presence without the user.

Since even today's most advanced virtual technologies are still imperfect and there is always a gap between the virtual experience and the realworld experiences (e.g., technological impediments in: visual-graphics fidelity, synchronization of multi-sensory perceptions, engineering of direct non-mediated haptic sensations), the user needs to "fill the gap" by creatively "filling in" some missing information or sensory signals, either by using cues from one sensory modality to compensate for impoverished and incomplete stimuli in another sensory modality, ${ }^{37}$ or/and by using his own creative imagination, previous knowledge etc. to construct from the entire set of presented stimuli and his internal knowledge a complete and coherent experience. ${ }^{38}$ Furthermore, a VE may contain also irrelevant and "noisy" information, and the user needs to concentrate only on the relevant cues, just like in the EFT. Thus, two processes are needed for achieving presence, ignoring the irrelevant cues and self-completion of the missing cues.

This is exactly the quality in which FI outperform FD-the cognitive ability to reorganize the immediately-perceived field and construct it according to their, previously acquired, internal 
knowledge. Hence, it is possible that in this study FI were far better able to cognitively reconstruct the haptic VE experiences more creatively, by using their own creative imagination and internal knowledge to fill in missing information, perceptions and sensations. As mentioned earlier, it has been shown already that higher involvement in the virtual experience leads to a greater sense of presence ${ }^{9}$; therefore, it is plausible to assume that this involvement in activating a cognitive compensatory mechanism that FI may have used, is one of the factors behind their enhanced sense of presence compared to the FD.

Furthermore, Creative Imagination is defined as "the ability to generate mental representations of objects, persons or events not immediately presented to the senses." ${ }^{\prime 39}$ One cannot avoid the similarity between this definition of creativity and the definition of Field Independency-the ability to overcome the organization of the field, break it up, and reconstruct it according to an internal model. ${ }^{14}$ Studies have also shown that FI tend to be more creative than FD ${ }^{40,41}$ and that creativity is positively correlated with the sense of presence. ${ }^{6,8}$ Hence, it may well be that it is a very similar (and overlapping) cognitive ability that manifests itself in both, creativity and field-independency, measures. And that the creative efforts, which FI employ in constructing their VE experiences, also enhance their sense of presence.

This proposed hypothesis on the relations between field dependency and the sense of presence in VE, may explain also other findings. In a recent study, ${ }^{42}$ participants learned about the human heart through a Web site with text accompanied by images of the heart in one of two formats, static images of the heart that could not be rotated (control condition), or a VE in which participants could rotate the heart to different viewpoints (the experimental condition). A recall test indicated that while the VE treatment did not improve FD performance, the FI VE group obtained a significantly higher score compared to the static-image group. According to the proposed relation between field dependency and presence it is plausible to speculate that the VE contribution to FI better performances had been achieved through the greater sense of (object) presence that the VE induced, since presence had been shown to improve memory and learning. ${ }^{43}$

In another experiment, ${ }^{44}$ extreme FD and FI males viewed an 8-min segment of film showing high-speed automobile travel. Half watched the film passively, and half watched while actively initiating leg movements in response to the car's expected turning direction. Skin conductance level was monitored from two nonpalmar sites, and the subjects reported the extent to which specific sensations were experienced. It was found that, although in the active viewing condition there was no difference, in the passive viewing condition, FI showed greater increases in skin conductance levels than FD, and electrodermal activity was associated with reported sensations of general discomfort and illusory motion. According to the hypothesis presented here, it may be that the cognitive mechanism behind these group differences in skin conductance and reported discomforts was the sense of presence which FI felt stronger than FD.

\section{Field-dependency, presence, and simulator sickness}

If the aforementioned hypothesis on the connection between field-dependency and presence is true, it may explain also other findings. Studies have found that visual-vertigo patients are significantly FD. ${ }^{45,46}$ Similarly, an experiment investigating the relationship between perceptual style and cue conflict induced by a large immobile swing inside of a moving room (an amusement park device), found that FD experienced the most discomfort in the swing when the room moved. ${ }^{47}$

There are also indications that those individuals who report more simulator sickness symptoms in $\mathrm{VE}$, report less presence than those who report fewer symptoms. ${ }^{4,48,49}$ Hence, it may be that it is the same cognitive style type-field dependencethat, on one hand, is more vulnerable to visual vertigo and simulator sickness, and on the other hand, experiences less presence in VE. Namely, those may actually be the two sides of the same coin: (a) a stronger visual dependency in the case of conflicting vestibular cues up to the point of vertigo and sickness symptoms, and (b) weaker cognitive reconstructing abilities in the presence of imperfect visual stimuli, which manifest itself in lower involvement and creativity, and result in a lower sense of presence. If so, field dependency scores may predict the extent of simulator sickness symptoms participants will exhibit, as FI may suffer less than FD. However, further studies with direct correlation between field dependency and simulator sickness are needed to determine that.

The hypothesis presented here does not contradict previous findings ${ }^{2}$ that people with visual dominance experienced the highest sense of presence. (a) Visual dominance is not necessarily identical with visual dependency. (b) In that study, participants were not exposed to an intersensory conflict between vision and the other senses (auditory and kinesthetic), they simply described their experiences with phrases either 
primarily visual, auditory or kinesthetic, whereas the visual vertigo and simulator sickness symptoms arise from the inter-sensory conflict-vision versus. vestibular cues. ${ }^{50,51}$ (c) Classification of participants into visually/auditory/kinesthetic dominance was based, in that study, on analyzing participants' subjective self descriptions about the VE experience, quantifying which sensory system they described more often, whereas classification of participants into visually dependents in the other study ${ }^{46}$ was based on a different and more objective measurethe RFT.

It is important to emphasize also the limitations of this study. First, it investigated a correlation between field-dependency and the sense of presence of an object in a non-immersive haptic VE. Thus, further studies are required to investigate the relations between field-dependency and the sense of being present in a place in fully immersive (360) VE. Furthermore, the number of participants in this study ( $n=18$, with gender equally balanced) does not permit broad conclusions. Another weakness is the measurement of presence by a post-experience, self-report and subjective questionnaire that may be methodologically circular and of very little scientific value. $^{52,53}$ Nevertheless, despite these limitations, the current results call for further studies to investigate more thoroughly the relationship between field dependency and the sense of presence in VE.

\section{ACKNOWLEDGMENTS}

This research was funded by the European project PRESENCIA-Presence: Research Encompassing Sensory Enhancement, Neuroscience and Cognition, with Interactive Applications. We also thank Mr. Gad Halevy for developing the game wth the virtual cubes that was used in this study.

\section{REFERENCES}

1. Slater, M., \& Usoh, M. (1993). Representation systems, perceptual position and presence in virtual environments. Presence: Teleoperators and Virtual Environments 2:221-233.

2. Slater, M., Usoh, M., \& Steed, A. (1994). Depth of presence in virtual environments. Presence: Teleoperators and Virtual Environments 3:130-144.

3. Slater, M., Usoh, M., \& Chrysanthou, Y. (1995). The influence of shadows on presence in immersive virtual environments. In: Goebel, M. (ed.), Virtual environments '95. New York: Springer Computer Science, pp. 8-21.
4. Witmer, B.G., \& Singer, M.J. (1998). Measuring presence in virtual environments: a presence questionnaire. Presence: Teleoperators and Virtual Environments 7:225-240.

5. Baños, R.M., Botella, C., Garcia-Palacios, A., et al. (1999). Psychological variables and reality judgment in virtual environments: the roles of absorption and dissociation. CyberPsychology $\mathcal{E}$ Behavior 2:143-148.

6. Sas, C., \& O'Hare, G.M.P. (2003). Presence equation: an investigation into cognitive factors underlying presence. Presence: Teleoperators \& Virtual Environments 12:523-527.

7. Sas, C., O'Hare, G.M.P., \& Reilly, R. (2004). Presence and task performance: an approach in the light of cognitive style. Cognition, Technology $\mathcal{E}$ Work 6:53-56.

8. Sas, C. (2004). Individual differences in virtual environments. Notes in Computer Science Lecture 3038: 1017-1024.

9. Sacau, A., Laarni, J., Ravaja, N., et al. (2005). The impact of personality factors on the experience of spatial presence. In: Slater, M. (ed.), Conference Proceedings of the $8^{\text {th }}$ International Workshop on Presence London, UK: University College, pp. 143-151.

10. Alsina, I., Carvallo, C., \& Gutiérrez Maldonado, J. (2005). Individual differences in the sense of presence. In: Slater, M. (ed.), Conference Proceedings of the $8^{\text {th }}$ International Workshop on Presence. London, UK: University College, pp. 133-142.

11. Witkin, H.A. Dyk, R.B., Faterson, H.F., et al. (1962). Psychological differentiation. New York: Wiley.

12. Witkin, H.A., \& Goodenough, D.R. (1981). Cognitive styles: essence and origins. Field dependece and independence. New York: International Universities Press.

13. Reger, G.M., McGee, J.S., van der Zaag, C., et al. (2003). A 3D virtual environment rod and frame test: the reliability and validity of four traditional scoring methods for older adults. Journal of Clinical \& Experimental Neuropsychology 25:1169-1177.

14. Witkin, H.A., Oltman, P.K., Raskyn, E., et al. (1971). A manual for the embedded figures tests. Palo Alto, CA: Consulting Psychologists Press.

15. Bell, E.G. (1955). Inner-directed and Other-directed attitudes. [Doctoral dissertation]. Yale University.

16. Gudjonsson, G.H. (1992). The psychology of interrogations, confessions and testimony. Chichester: Wiley, UK.

17. Blagrove, M., Cole-Morgan, D., \& Lambe, H. (1994). Interrogative suggestibility: the effects of sleep deprivation and relationship with field-dependence. Applied Cognitive Psychology 8:169-179.

18. Manning, L. (1991). Objective and Subjective Factors in Field Dependence-Independence. In: Wapner, S., \& Demick, J. (eds.), Field dependence-independence: $\operatorname{cog}$ nitive style across the life span. Hillsdale: NJ: Lawrence Erlbaum Assosiates, pp. 85-103.

19. Goldenberg, N., \& Wachtel, P. (1973). Hypnotizability and cognitive controls. International Journal of Clinical E Experimental Hypnosis 21:298-304. 
20. Gates, D.W. (1971). Verbal conditioning, transfer and operant level "speech style" as functions of cognitive style. [Doctoral dissertation]. City University of New York.

21. Melancon, J.G., \& Thompson, B. (1989). Measurement characteristics of the finding embedded figures test. Psychology in the Schools 26:69-78.

22. Elliot, R. (1961). Interrelationships among measures of field independence, ability and personality traits. Journal of Abnormal and Social Psychology 63:27-38.

23. Hergovich, A. (2003). Field dependence, suggestibility and belief in paranormal phenomena. Personality and Individual Differences 34:195-209.

24. Nisbett, R.E., \& Masuda, T. (2003). Culture and point of view. Proceedings of the National Academy of Sciences of the U.S.A. 100:11163-11170.

25. Ji, L., Peng, K., \& Nisbett, R.E. (2000). Culture, control, and perception of relationships in the environment. Journal of Personality \& Social Psychology 78:943-955.

26. Kitayama, S., Duffy, S., Kawamura, T., et al. (2003). Perceiving an object and its context in different cultures: a cultural look at new look. Psychological Science 14:201-206.

27. Nisbett, R.E., \& Miyamoto, Y. (2005). The influence of culture: holistic versus analytic perception. Trends in Cognitive Science 9:467-473.

28. Heine, S., Lehman, D., Markus, H., et al. (1999). Is there a universal need for positive self-regard? Psychological Review 106:766-794.

29. Triandis, H. (1995). Individualism and collectivism. Boulder, CO: Westview.

30. Korchin, S.J. (1986). Field Dependence, Personality Theory and Clinical Research. In Bertini, M., Pizzamiglio, L. \& Wapner, S. (eds.), Field dependence in psychological theory, research and application. Hillsdale, NJ: Lawrence Erlbaum Assosiates, pp. 45-56.

31. Oltman, P.K. (1986). Psychological differentiation theory in social and cross-cultural psychology. In: Bertini, M., Pizzamiglio, L. \& Wapner, S. (eds.), Field dependence in psychological theory, research and application. Hillsdale, NJ: Lawrence Erlbaum Assosiates, pp. 85-91.

32. Reiner, M. (2004). The role of haptics in immersive telecommunication environments. IEEE Transactions on Circuits and Systems for Video Technology 14: 392-401.

33. Stevens, B., \& Jerrams-Smith, J. (2001). The sense of object-presence with projection-augmented models. Lecture Notes in Computer Science 2058:73-75.

34. Stevens, B., Jerrams-Smith, J., Heathcote, D., et al. (2002). Putting the virtual into reality: assessing objectpresence with projection-augmented models. Presence: Teleoperators and Virtual Environments 11:79-92.

35. Demick, J. (1991). Organismic factors in field dependence-independence: gender, personality, psychopathology. In: Wapner, S., \& Demick, J. (eds.), Field dependence-independence: cognitive style across the life span. Hillsdale, NJ: Lawrence Erlbaum Assosiates. pp. 209-243.
36. Meehan, M., Insko, B., Whitton, M.C., et al. (2002). Physiological measures of presence in stressful virtual environments. Presented at the $29^{\text {th }}$ Annual Conference on Computer Graphics and Interactive Techniques (SIGGRAPH), San Antonio, TX.

37. Biocca, F., Kim, J., \& Choi, Y. (2001). Visual touch in virtual environments: an exploratory study of presence, multimodal interfaces, and cross-modal sensory illusions. Presence: Teleoperators \& Virtual Environments 10:247-266.

38. Jansson, G. (2001). The potential importance of perceptual filling-in for haptic perception of virtual object form. In: Baber, C., Faint, M., Wall, S., et al. (eds.), Conference Proceedings of Eurohaptics 2001 (Educational Technology Research Papers), Birmingham, UK: University of Birmingham, pp. 72-75.

39. Singer, J. (2000). Imagination. In: Kazdin, A. (ed.), Encyclopedia of Psychology, Vol. 4. New York: Oxford University Press, pp. 227-230.

40. Rastogi, M. (1987). A study of cognitive style in relation to intelligence and creativity. Journal of Personality $\mathcal{E}$ Clinical Studies 3:161-164.

41. Noppe, L.D. (1985). The relationship of formal thought and cognitive styles to creativity. Journal of Creative Behavior 19:88-96.

42. Ogle, T. (2002). The effects of virtual environments on recall in participants of differing levels of field dependence. [Doctoral dissertation]. Virginia Polytechnic and State University, Blacksburg, VA.

43. Dinh, H.G., Walker, N., Hodges, L.F., et al. (1999). Evaluating the importance of multi-sensory input on memory and the sense of presence in virtual environments. In: Rosenblum, L. Astheimer, P., \& Teichmann, D. (eds.), Proceedings of the IEEE Virtual Reality '99 Conference. Los Alamitos, CA: IEEE Computer Society Press, pp. 222-228.

44. Alexander, R.A., \& Barrett, G.V. (1975). Relationship between perceptual style and responses to visual motion under active and passive viewing conditions. Journal of Applied Psychology 60:507-512.

45. Bronstein, A.M. (2002). Under-rated neuro-otological symptoms: Hoffman and Brookler 1978 revisited. British Medical Bulletin 63:213-221.

46. Guerraz, M., Yardley, L., Bertholon, P., et al. (2001). Visual vertigo: symptom assessment, spatial orientation and postural control. Brain 124:1646-1656.

47. Barrett, G.V., Thornton, C.L., \& Cabe, P.A. (1970). Cue conflict related to perceptual style. Journal of Applied Psychology 54:258-264.

48. Kingdon, K., Stanney, K.M., \& Kennedy, R.S. (2001). Extreme responses to virtual environment exposure. In: Proceedings of the $45^{\text {th }}$ annual human factors and ergonomics society meeting. Minneapolis/St. Paul, MN, pp. 1906-1910.

49. Sadowski, W., \& Stanney, K.M. (2002). Presence in virtual environments. In: K.M. Stanney (ed.), Handbook of virtual environments: design, implementation, 
and applications Mahwah, NJ: Lawrence Erlbaum Associates, pp. 791-806.

50. Draper, M.H., Viire, E.S., Furness, T.A., et al. (2001). Effects of image scale and system time delay on simulator sickness within head-coupled virtual environments. Human Factors 43:129-146.

51. Duh, H.B.L., Parker, D.E., Philips, J.O., et al. (2004). "Conflicting" motion cues to the visual and vestibular self-motion systems around $0.06 \mathrm{~Hz}$ evoke simulator sickness. Human Factors 46:142-153.

52. Slater, M. (2004). How colorful was your day? Why questionnaires cannot assess presence in virtual environments. Presence: Teleoperators and Virtual Environments 13:484-493.
53. Sanchez-Vives, M.V., \& Slater, M. (2005). From presence to consciousness through virtual reality. Nature Reviews: Neuroscience 6:332-339.

Address reprint requests to: David Hecht

Department of Behavioral Biology The Touch Laboratory

Gutwirth Building, Technion City Haifa, 32000, Israel

E-mail: davidh@tx.technion.ac.il 